\title{
Propuesta de control de asistencia y deserción estudiantil en la Universidad Tecnológica de El Salvador
}

\author{
Proposal for attendance and retention control at \\ Universidad Tecnológica de El Salvador
}

ISSN 2071-8748

E-ISSN 2218-3345

(c) (1) (2)

BY NC SA

DOI: https://doi.org/10.5377/entorno.v0i68.8444

URI: http://hdl.handle.net/11298/1124

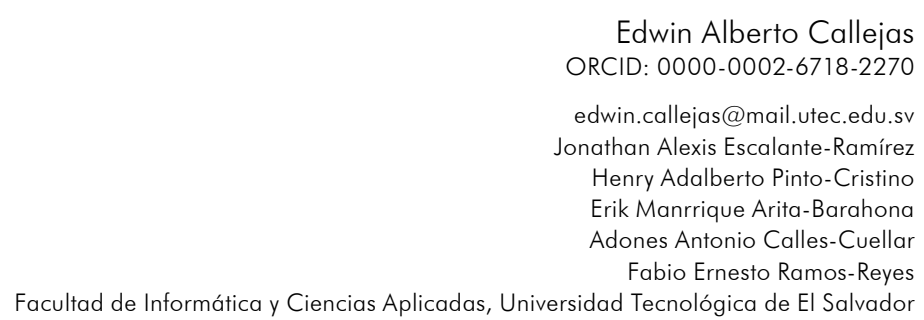

Recibido: 4 de mayo de 2019

Aprobado: 13 de septiembre de 2019

\section{Resumen}

El presente proyecto de investigación describe una propuesta de solución tecnológica para el control de asistencia y deserción estudiantil en la Universidad Tecnológica de El Salvador, a través de una herramienta que facilite la realización de las actividades administrativas y académicas de apoyo al docente y la institución para el monitoreo de los estudiantes que asisten o no a la universidad. El estudio permitirá optimizar los procesos de administración de control de asistencia y deserción estudiantil que la universidad ejecuta y complementan la gestión académica del docente sobre sus estudiantes. Se diseña un modelo que hace uso de los recursos actuales de la institución, se desarrolla y valida la herramienta tecnológica para apoyar el modelo propuesto que es elaborado por un grupo de estudiantes, utilizando el lenguaje de programación de PHP para el diseño e implementación de interfaces dinámicas y amigables que

\section{Abstract}

This research Project describes a proposal for a technological solution for the control of attendance and retention at Universidad Tecnológica de El Salvador, by means of a tool that facilitates the administrative and academic work to support faculty members and the institution itself, in order to monitor student attendance. This study will allow for the optimization of the attendance control processes and the retention processes that the university executes and which complement the academic management of the faculty members over their students. A model using the current resources of the institution has been designed; the technological tool has been developed and validated so as to provide support to the proposed model; said model was created by a group of students who used PHP programming language for the design and implementation of dynamic and user-friendly interfaces that will allow the user to 
permitirá al usuario un manejo fácil a través de dispositivos móviles y una herramienta de administración web.

\section{Palabras clave}

Asistencia a la universidad - Universidad Tecnológica de El Salvador - modelos. Deserción universitaria Universidad Tecnológica de El Salvador - modelos. tecnología web - tecnología móvil

\section{Introducción}

La Universidad Tecnológica de El Salvador (Utec) se fundó el 12 de junio de 1981, en San Salvador, como propiedad de una sociedad privada. El campus de la Universidad se encuentra ubicado en San Salvador y cuenta, después de 40 años desde su fundación, con una infraestructura física de más de 23 edificaciones donde funcionan aulas, auditorios, bibliotecas, laboratorios especializados, oficinas administrativas, entre otras. Las facultades de la Universidad son las siguientes: Facultad de Informática y Ciencias Aplicadas, Facultad de Ciencias Empresariales, Facultad de Derecho, Facultad de Ciencias Sociales y Facultad de Maestrías y Estudios de Posgrado, las cuales atienden una población estudiantil de casi 22.500 estudiantes y unos 700 docentes. En la gestión académica administrativa, la Universidad mantiene un proceso de monitoreo de la asistencia en periodos clave por cada asignatura, sin embargo, existe una serie de inconvenientes que dificulta obtener información relevante y oportuna para apoyar los procesos de toma de decisión en el control de la asistencia y el monitoreo de la deserción por la alta cantidad de estudiantes. Además, la infraestructura tecnológica instalada no está orientada específicamente a apoyar el proceso bajo estudio, sino, más bien, a otros servicios que la población universitaria requiere, como tareas académicas, administrativas e investigación.

La infraestructura tecnológica de la Universidad debe de responder a las necesidades que los procesos institucionales demanden, para mantenerse siempre como líder al brindar servicios educativos de calidad y posicionarse como un referente en el ámbito educativo a escala regional en el uso de tecnología. Se destaca el control de la asistencia y la deserción estudiantil como una necesidad imperante para apoyarse en la toma de decisiones, mediante uso de herramientas tecnológicas para obtener información confiable, rápida y eficazmente. easily manage it by using mobile devices and a tool that is managed via web.

\section{Keywords}

College Attendance - Universidad Tecnológica de El Salvador - models. College retention/drop outs Universidad Tecnológica de El Salvador - models. - web technologies - mobile technology.

La deserción estudiantil (Himmel, 2002) es un fenómeno que se puede categorizar de muchas maneras (Elias, 2008). Sin embargo, para efectos de nuestro estudio, se hace referencia al abandono prematuro de su programa de estudios, no concluyendo su grado académico; esta deserción puede ser voluntaria o involuntaria, en este segmento. La institución debe de redoblar esfuerzos para identificar las causas que originarían una posible deserción mediante el control de asistencia del estudiante.

El control de asistencia estudiantil (Pérez, 2004) es un mecanismo que se utiliza de soporte a tareas a administrativas y para supervisar la asistencia de los estudiantes a clases, entre otras, con el propósito de monitorear el grado de deserción estudiantil en la asignatura que tenga como consecuencia el abandono de los estudios; las causas podrían ser pedagógicas, financieras, por horarios o accesibilidad, entre otros (Quispe, 2010).

El proceso de control de asistencia y deserción estudiantil ha evolucionado de forma permanente en la Utec; siempre se han ido superando las deficiencias para realizar este requerimiento (Rodríguez, 2015). Se ha pasado de procesos en los que no se consideraba su impacto para la toma de decisiones institucionales, procesos reactivos donde el docente a tiempo completo realizaba dicha tarea suspendiendo sus actividades administrativas académicas, a procesos sistemáticos para cubrir la realización de la tarea con los recursos disponibles de docentes y la incorporación de estudiantes para su realización, por mencionar algunos puntos de mejora. En los años recientes se ha incorporado a estudiantes en horas sociales como apoyo para la recolección de datos y su posterior procesamiento por cada una de las unidades académicas.

El control de asistencia estudiantil, en la Utec, actualmente es un proceso manual e inexacto, lento y poco efectivo. Se 
selecciona a un grupo de estudiantes en servicios de horas sociales y se les asigna un horario de conteo en el que visitan las aulas donde se imparten asignaturas en ese horario en todo el campus donde se desplazan a cada aula para anotar a mano a cada estudiante presente en un formato diseñado para tal fin. Cuando el estudiante finaliza el recorrido del grupo de asignaturas que se le ha asignado, entrega la información al responsable del horario para que realice la carga de datos. Luego, este tiene que llenar manualmente un formulario para reportar dichas cantidades para ser consolidadas, por lo tanto, las cifras que maneja la institución podrían ser incorrectas, ya que no todos los que están presentes en el aula durante el conteo podrían ser estudiantes inscritos en la signatura que se está impartiendo a esa hora, y a veces ni inscritos en la universidad, por lo tanto, no se maneja un control adecuado al ingreso en las aulas de clases.

Algunos problemas que se detectan actualmente son los siguientes:

- Información no centralizada: la información pasa por muchas manos antes de ser procesada, lo cual puede implicar que la información sea manipulada y esto afecte los resultados en los datos finales.

- Datos poco confiables: por la forma y en el tiempo en que se recolectan los datos, estos son poco confiables.

- Gasto de recurso como papel: para poder realizar la recolección de datos, se usa todos los días papel y tinta; es un gasto que se tiene actualmente. Esto representa un gasto para la universidad, además de una sub utilización de recursos

- Inversión de tiempo: para poder tener los datos, actualmente se necesita invertir mucho tiempo, pues implica que una persona visite las aulas una por una y pasar un mínimo de 3 minutos por cada aula para realizar un conteo más o menos exacto.

- Reportes deficientes: el generar los reportes actualmente requiere de la intervención de muchas personas y de muchos pasos, esto genera pérdida de tiempo y poca confiabilidad en la información. Generar más información de apoyo a la toma decisiones adicionales, se vuelve una tarea compleja, por la cantidad de recursos que esta tarea demandaría.

El proceso no se encuentra documentado, por lo que se deja a discrecionalidad de cada Facultad la forma en que se realiza y mejorar los plazos de entrega de la información para lograr hacer análisis de variables oportunos de apoyo en la toma de decisiones, por lo que solucionar este problema con la incorporación de tecnología será una ventaja para la institución, que le permitirá ahorrar tiempo, esfuerzo, consumo de recursos, estrés laboral y proporcionará información más completa, fiable y oportuna para el apoyo en la toma de decisiones.

\section{Metodología}

La metodología utilizada para desarrollar la presente investigación y en la elaboración del prototipo de control de asistencia y deserción estudiantil fue un modelo cuasiexperimental que parte de usar técnicas de recolección de información como la observación, las entrevistas, encuestas y de la experimentación de los resultados mediante la simulación de procesos para validar los resultados obtenidos, con el uso del prototipo propuesto. Con la información recolectada sobre el proceso, se analizaron y determinaron los requerimientos de información necesarios para poder diseñar el nuevo flujo de información con base en la capacidad tecnológica de hardware y software que tiene la institución. Asimismo, se diseñaron los nuevos prototipos tecnológicos que se elaborarán para lograr crear un ecosistema funcional donde pueda operar la herramienta tecnológica que permita controlar la asistencia y disminuir la deserción estudiantil. El marco de trabajo que nos permitió implementar la metodología fue SCRUM a través del ciclo de vida de desarrollo de software implementado implementando los valores establecidos en el manifiesto ágil (Ángel Medinilla, 2018)

\section{Diseño del modelo}

El modelo que se diseñó para hacer funcional la herramienta tecnológica propuesta fue una arquitectura híbrida, que facilitará la interoperabilidad entre los diferentes componentes de la solución de la siguiente manera:

La identificación de los actores como potenciales usuarios permitió generar los niveles de privilegios de acceso que se tendrá a la información y a los procesos que se les asignará a cada uno de ellos, entre los que podemos mencionar a los estudiantes, docentes, directores, decanos, vicerrector, rector y administrador, así como determinar el nivel de confidencialidad, seguridad y consumo de recursos necesarios para ser considerados en la herramienta tecnológica y definir los criterios de usabilidad, escalabilidad y de rendimiento que faciliten trabajar óptima y ágilmente. 
Figura 1. Diagrama arquitectónico de propuesta de control de asistencia y deserción estudiantil

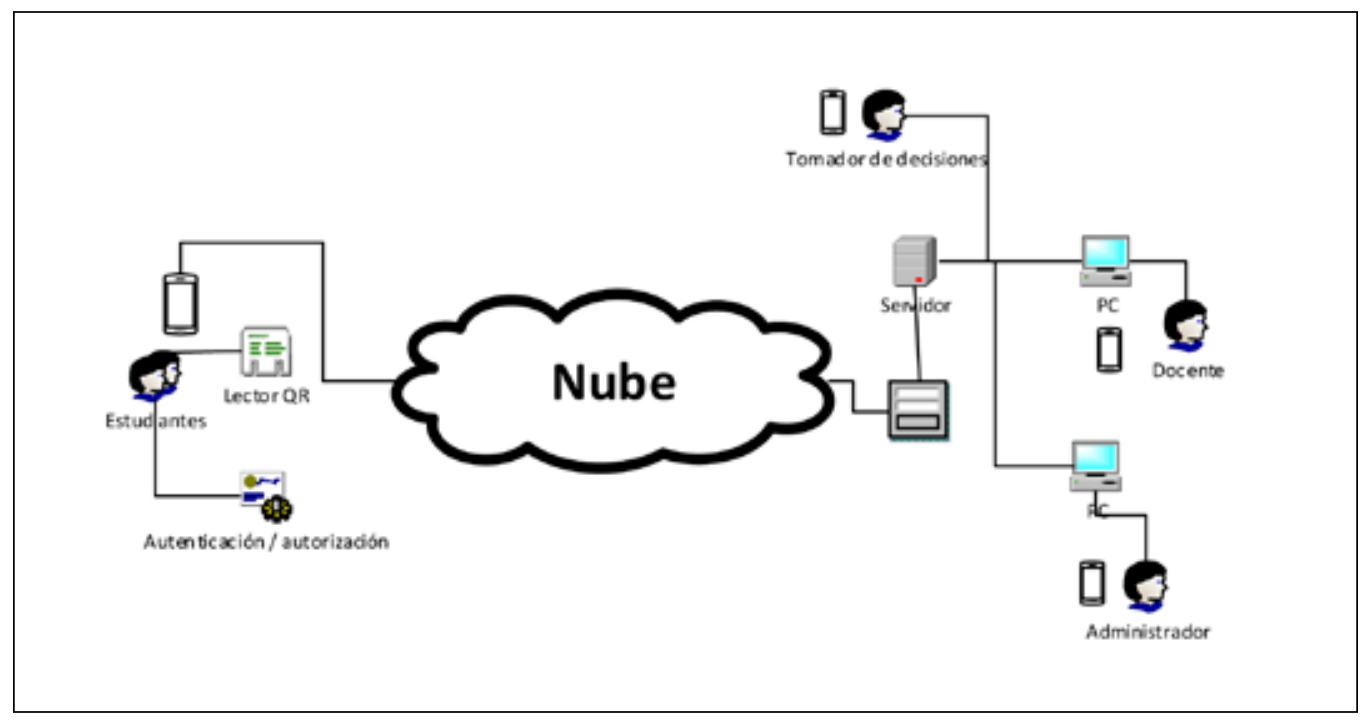

Como se observa en la figura 1, la propuesta tecnológica para el control de asistencia y deserción estudiantil involucra configurar un ecosistema de tecnologías para operar en un sistema de información que pueda interoperar con fuentes de información mediante diferentes dispositivos (Mashup, 2018). Entre las tecnologías se mencionan: un sistema informático web elaborado en lenguaje de programación PHP, el cual permitirá diseñar interfaces accesibles y funcionales para la implantación de procesos que sean consumidos mediante smartphones, tablets, computadoras o servicios web; por medio de un navegador web, consumir sus servicios. Los usuarios del sistema informático web serán el administrador y los usuarios consumidores de la información que el sistema informático procesa. La aplicación móvil que se encargará de consumir servicios web facilitará consultar la información generada por el sistema informático, como además facilitar el acceso para el registro de la asistencia. El lenguaje de programación de las aplicaciones móviles será diseñado para operar en sistema operativo Android y iOS.

La aplicación móvil ofrecerá la funcionalidad de registro de la asistencia, facilitando la operación de leer un código QR (Wikipedia, 2019) que contiene la información de las asignaturas que se imparten en un aula y el intervalo de horario en que se imparte, de tal manera que, cuando un estudiante escanee el código, el sistema detecte la asignatura en que se quiere registrar la asistencia y permita transmitir la asistencia a clases del estudiante si está inscrito en la asignatura, requiriendo que, para que el proceso se ejecute satisfactoriamente, el estudiante lo haga mediante un dispositivo móvil con acceso a la red WiFi de la Universidad y que posea el certificado digital institucional para conectarse a internet. Tanto el docente como el estudiante harán uso de la aplicación móvil. Por tal motivo, el proceso de autenticación y autorización se ha diseñado considerando métodos internos, accesibles e innovadores con la tecnología de la institución disponible.

La base de datos se implementará en el proveedor MySQL, estará centralizada en un servidor que será accesible mediante la nube de la Utec y desde donde se accederá a los datos para procesarlos y almacenarlos. Todo lo antes mencionado entra en las nuevas tecnologías y por ende en el tema de las tecnologías de la información y la comunicación, cuya base se centra en los campos de la informática, la microelectrónica y las telecomunicaciones para dar paso a la creación de nuevas formas de comunicación. Cuando se registra la asistencia por medio del QR, se está haciendo uso de tecnologías como la del smartphone, que la guarda en un sistema informático web; y de ahí una persona encargada verifica toda la información capturada. Las características del hardware necesario para utilizar la solución propuesta es la mostrada en las tablas 1 y 2. 
Tabla 1. Requisitos mínimos para App de asistencia

\begin{tabular}{|l|l|}
\hline Característica & Descripción \\
\hline S.O & Android 4.o o superior \\
\hline Cámara & 2.0 mega pixeles o sup. \\
\hline Conectividad & Intranet \\
\hline
\end{tabular}

Tabla 2. Requisitos mínimos para docente y administrador

\begin{tabular}{|l|l|}
\hline Característica & Descripción \\
\hline SO & $\begin{array}{l}\text { Windows 7 o superior, } \\
\text { Android, iOS }\end{array}$ \\
\hline Almacenamiento & $1 \mathrm{~GB}$ \\
\hline RAM & $512 \mathrm{Mb}$ \\
\hline Navegador & $\begin{array}{l}\text { Chrome, Mozilla Firefox, } \\
\text { Internet Explorer }\end{array}$ \\
\hline
\end{tabular}

\section{Descripción del escenario}

Hacia una transformación digital UTEC: Propuesta de control de asistencia y deserción estudiantil. La propuesta elaborada consiste en cargar en una base de datos la distribución de la carga académica de la Universidad, que contiene el aula, docente y horario en el que se imparten las asignaturas, en un módulo restringido al administrador con el propósito de generar un código QR que contiene la distribución de las asignaturas que se imparten en un horario específico por aula. Este QR se imprimirá en un sticker que se colocará en cada aula, para que el estudiante registre su asistencia mediante un dispositivo móvil. En la figura 2 se muestra la interfaz del administrador de la propuesta, y los elementos se describen a continuación:

1. Carga de datos del ciclo: son los datos maestros del sistema.

2. Gestión de datos cargados: sirve para ver la información que se ha cargado en el sistema, carga académica.
3. Códigos QR para aulas: sirve para generar los códigos QR con los cuales los estudiantes generarán la asistencia.

4. Consultar carga académica por Facultad: ver la información de la carga académica por Facultad.

5. Consultar asistencia por bloque de horarios: ver la asistencia por bloque de horario en específico.

6. Consulta asistencia por edificio: ver la asistencia por cada edificio en general.

7. Consultar asistencia por nivel de edificio: ver asistencia por edificio pero en un nivel en específico.

8- Tendencia de asistencia por edificio: ver la tendencia de la asistencia en un edificio en específico.

9- Tendencia de asistencia por bloque de horario: ver la tendencia de asistencia en un bloque de horario en específico.

10. Gestión de usuarios: gestionar la información de los usuarios del sistema con tareas de agregar, modificar y eliminar usuarios. 


\section{Figura 2. Interfaz del administrador de gestión de la carga académica}

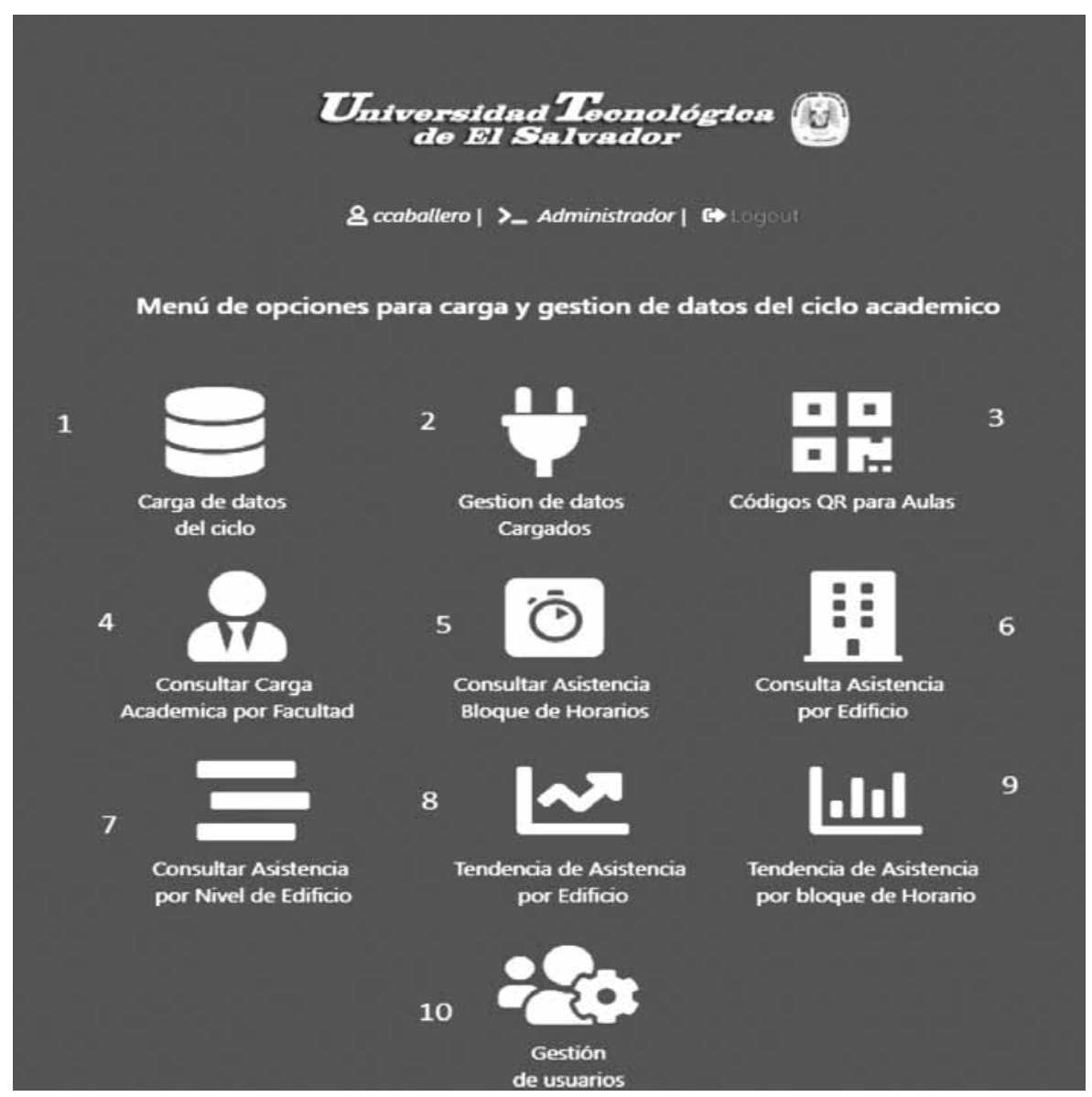

La propuesta incluye una aplicación móvil donde el estudiante se deberá registrar con sus credenciales, que le habilitará la cámara para escanear el código QR en el aula. El sistema buscará su horario y las clases en las que está inscrito y comparará la hora del sistema con el horario del estudiante, mostrando así una ventana emergente preguntando al alumno si quiere registrar su asistencia a la clase asignada a esa esa hora en el aula, el estudiante solo confirmará; y de esa forma estará registrando su asistencia de ese día, en una asignatura determinada; así la Universidad tendrá un control efectivo y exacto de los alumnos que asisten a dicha clase.
1. El usuario deberá ingresar con su número de carné

2. La contraseña es el mismo número del carné

3. El botón de login le permitirá acceder al lector QR

4. Nombre de la empresa desarrolladora

5. Logo y nombre de la Universidad Tecnológica de El Salvador

6. Cámara lectora de $Q R$

7. Botón para cerrar sesión

8. Formulario del registro de asistencia, el cual no se puede modificar

9. Botón para registrar la asistencia 
Figura 3. Interfaz de aplicación móvil para que el estudiante registre su asistencia
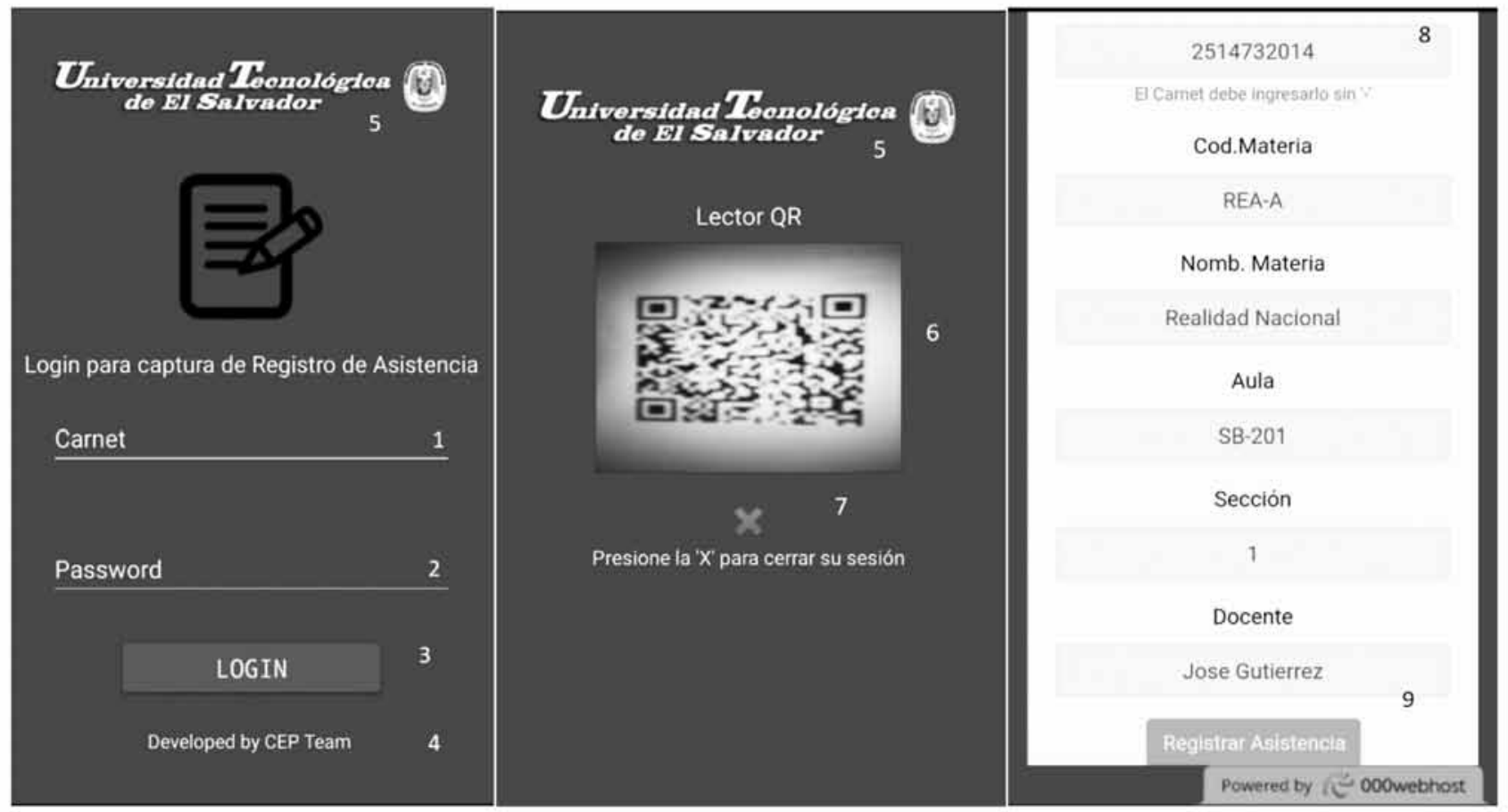

Para los docentes, se creó una interfaz dinámica y amigable, en entorno web, que le permitirá un control total de la asistencia una vez que registre los estudiantes inscritos en su asignatura. El sistema les permitirá graficar y de esta manera monitorear la asistencia de los estudiantes a lo largo del ciclo para identificar las altas y bajas. De igual manera, el sistema generará reportes diarios de los estudiantes que asisten a clases. Esto permitirá al docente un mejor control en las actividades evaluadas que se ejecuten por día.

Figura 4. Interfaz web de acceso al docente, para que pueda llevar el control de asistencia de sus estudiantes
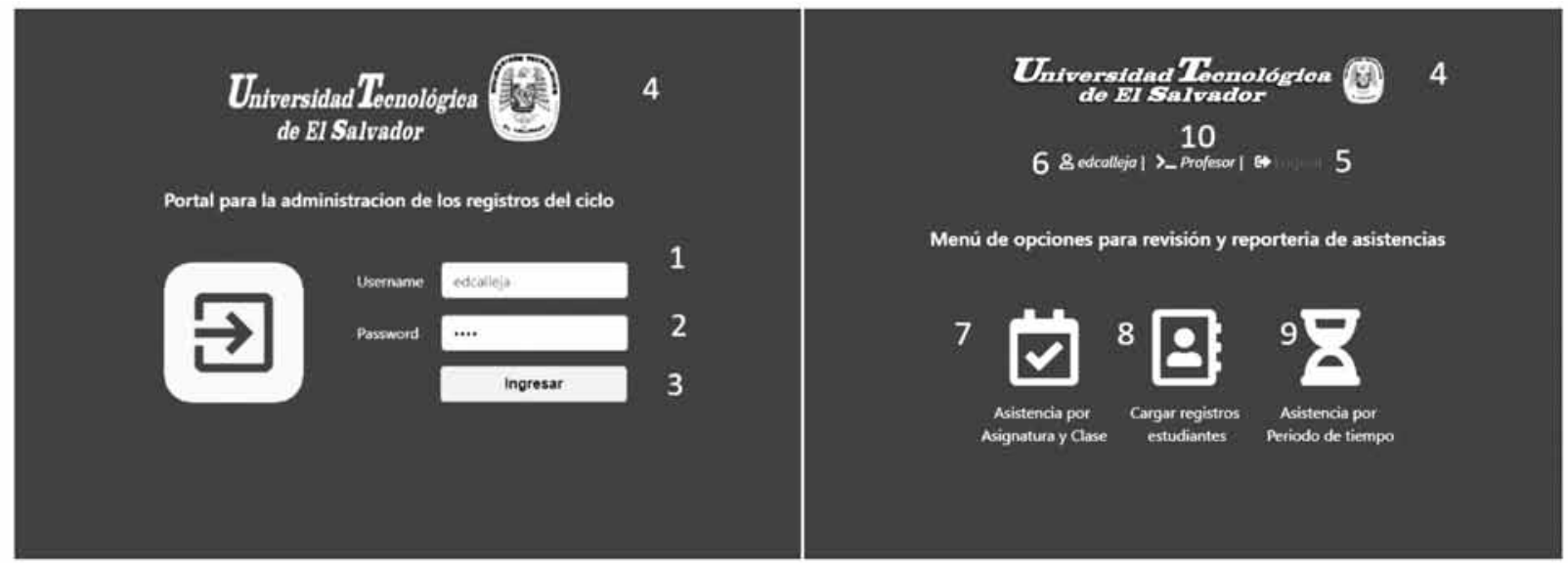
El proceso de operación de la interfaz de gestión de información, por parte del docente, se describe a continuación:

1. El docente digitará su usuario

2. El docente digitará su contraseña

3. El botón Ingresar lo va a transferir al siguiente menú

4. Logo y nombre de la Universidad

5. Botón para salir del sistema

6. Usuario y nivel de privilegios

7. Icono para revisar la asistencia diaria

8. Icono para la carga de estudiantes

9. Icono para ver las gráficas por periodo

10. Botón para regresar al menú

\section{DISCUSIÓN}

Con el desarrollo de los diferentes módulos de la solución, se ha dado respuesta a lo que en un inicio fue el problema por resolver: controlar la asistencia y la deserción estudiantil. La solución propuesta de Control de Asistencia de la Universidad Tecnológica de El Salvador, permitirá que los estudiantes registren su asistencia en una clase determinada, pero también permite al docente mostrar indicadores que le facilitarán tener datos confiables y al instante con respecto a su grupo de clases; de la misma manera los administradores académicos, como catedráticos, directores, decanos y vicerrector Académico, tendrán información que les ayudará a la toma de decisiones.

Figura 6. Interfaz de control de asistencia
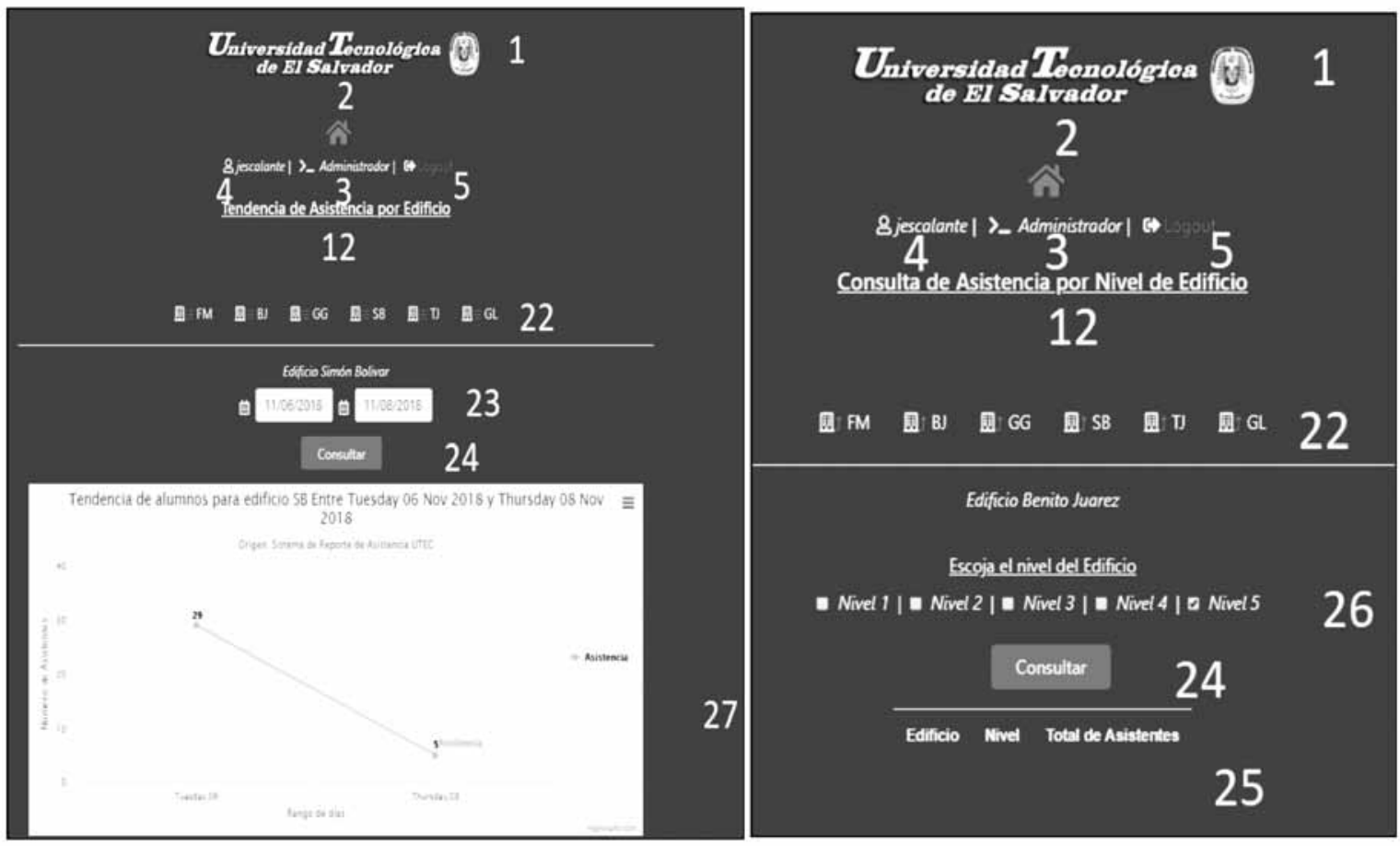

La decisión de proponer un sistema que implementara un lector $\mathrm{QR}$, fue porque la mayoría de la población estudiantil de la Utec, posee dispositivos móviles inteligentes, y esto ofrece una gran facilidad en su implementación. Sin embargo, cuando un estudiante no cuente con su dispositivo móvil podrá autenticarse en otro dispositivo de un compañero de clase y así poder registrar su asistencia. 
Figura 5. Control de estadísticos, vista de gestor académico

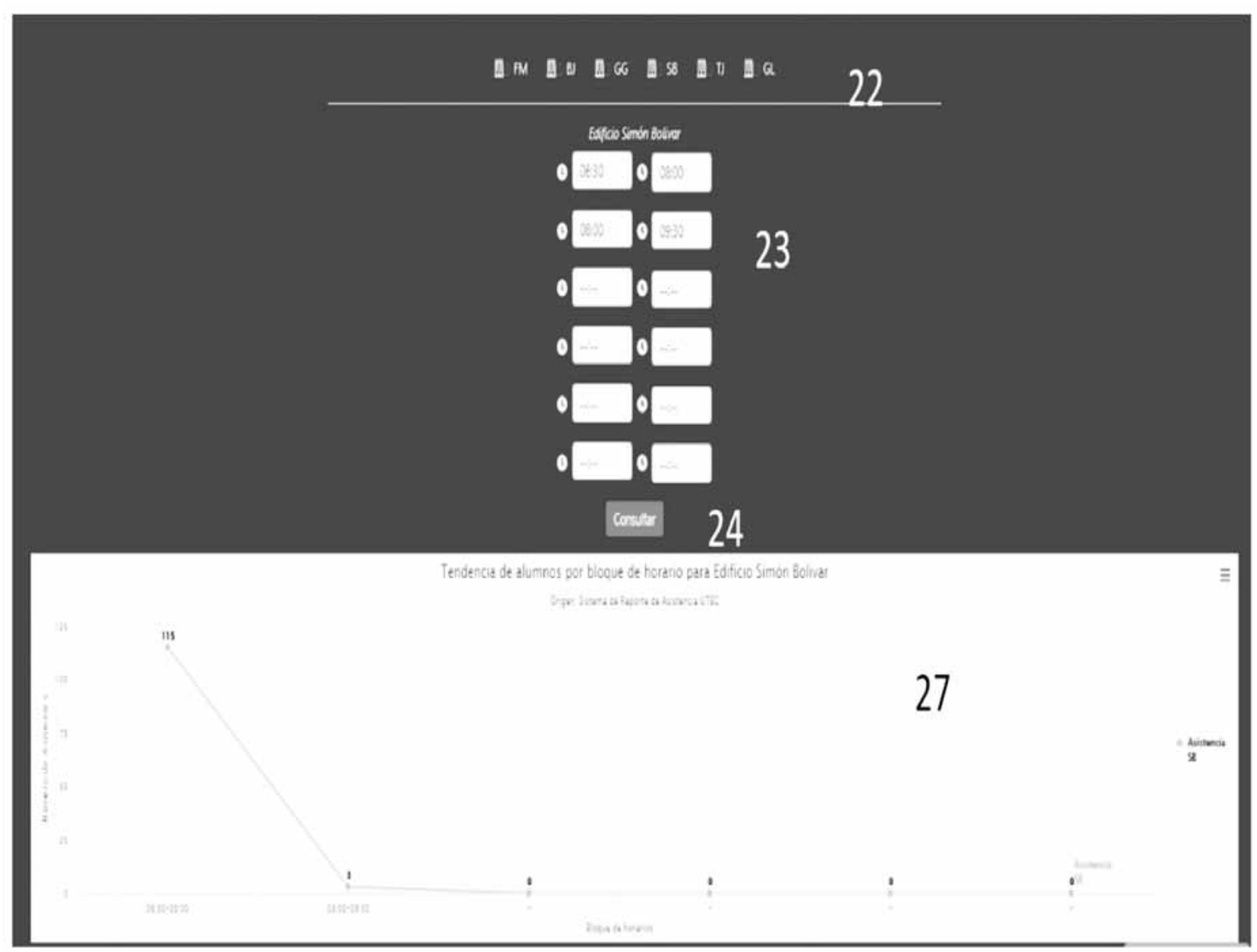

Entre los trabajos futuros que se pueden desarrollar a partir de la automatización de la asistencia de los alumnos de la Utec se encuentran los siguientes:

- Integración del sistema: el sistema de control de asistencia se puede integrar con el sistema de matrícula y la planificación de carga académica, y en un futuro se evitará estar cargando los datos cada ciclo para poder llevar el control de asistencia de los estudiantes, esto permitiría que, una vez el estudiante inscriba sus asignaturas, el sistema de control de asistencia pueda reconocer esos datos y estar listo para funcionar.

- Implementación de pantallas digitales en lugar de stickers para disponer del código QR en las aulas: con la implementación de pantallas, la Universidad estaría contribuyendo al medio ambiente con el ahorro en el uso de papel y tinta que se estaría usando cada vez que el sticker sufra de deterioro. Estas pantallas la universidad podrá usarlas para mostrar anuncios a los alumnos una vez haya pasado el tiempo para registrar la asistencia correspondiente a una clase determinada.

- Mejorar la infraestructura WiFi: facilitar la comunicación de datos, ya que actualmente hay puntos de los edificios donde la conectividad a la red WiFi de la Utec no tiene mayor intensidad y en momentos con mayor transmisión de datos puede fallar

\section{Conclusiones}

El presente proyecto de investigación de control de asistencia y deserción estudiantil generó una propuesta de mecanización del control de asistencia de los estudiantes de la Utec que puede impactar positivamente la manera en que se realiza dicho control y disminuir la deserción estudiantil, esto debido a que se necesitan pocos recursos para 
poder implantar la propuesta, logrando que la información que el sistema facilite a docentes, directores, decanos y vicerrectores para la toma de decisiones sea confiable y oportuna. El sistema cuenta con sus propias características que permiten aprovechar las funcionalidades tanto en el aspecto de presentación dinámica y amigable para el usuario final como en el de la eficiencia en el almacenamiento y manejo de contenido.

Los beneficios que se obtendrán con la implementación del Sistema de Control de Asistencia de la Universidad Tecnológica de El Salvador son los siguientes:

- Información centralizada: los datos podrán ser consultados desde cualquier dispositivo con conexión a la red interna de la Universidad, teniendo las credenciales necesarias.

- Datos confiables: el conteo manual será parte del pasado; usar tecnología computarizada permitirá registrar datos confiables con respecto a la asistencia a clases de los estudiantes.

- Ahorro considerable de papel y tinta: ya no se usará papel cada día para anotar la asistencia de los estudiantes a clases, esto se traducirá en ahorro económico para la institución.

- Reducción del tiempo dedicado a obtener los datos de la asistencia de los estudiantes: en el mismo instante en que el alumno registre su asistencia, ese dato lo podrá ver vía internet tanto el docente como el rector de la universidad sin intermediarios.

- Disponibilidad de reportes: el sistema mostrará una serie de reportes y gráficas de los diferentes datos respectivos a la asistencia de los alumnos.

- Reasignación de actividades de las personas que actualmente colaboran con la recolección de la asistencia: las personas que día a día van por las aulas recogiendo los datos podrán invertir su tiempo en otras actividades que beneficien tanto a ellos como a la Universidad.
Referencias

Abarca Rodríguez, A. y Sánchez Vindas, M. A. (julio, 2005). La deserción estudiantil en la educación superior: El caso de la Universidad de Costa Rica. Actualidades Investigativas en Educación, 5. Recuperado de https://revistas.ucr.ac.cr/index.php/aie/article/ view/9186/17625

Cordero Cordero, T. (diciembre, 2008). La opinión de un grupo de docentes sobre la deserción escolar. Explorando sobre sus actuaciones en el contexto institucional. Actualidades investigativas en Educacion, 8 (3), 1-33. Recuperado de https://revistas.ucr.ac.cr/index.php/ aie/article/ view/9354/17834

Elías Andreu, M. (2008). Los abandonos universitarios: Retos ante el espacio Europeo de educación superior. Estudios Sobre Educación, (15), 101-121. Recuperado de https://www.unav.edu/pu blicaciones/revistas/ index.php/estudios-sobre-educacion/article/ view/23447/19405

Fundación Wikimedia, Inc. (2018). Mashup (aplicación web híbrida). Recuperado de https://es.wikipedia.org/wiki/ Mashup_(aplicación_web_híbrida)

Fundación Wikimedia, Inc. (2019). Código QR. Recuperado de https://es.wikipedia.org/wiki/ Código_QR

Himmel, E. (2002). Modelos de análisis para la deserción estudiantil en la educación superior. Calidad de la Educación, (17), 91-108. doi: http://dx.doi. org/10.31619/caledu.n17.409

Medinilla, A., Giné, A. y Gómez, E. (2018). Manifiesto por el desarrollo ágil de Software. Recuperado de http:// agilemanifesto.org/iso/es/manifesto.html

Pérez, J. y Graell, S. (2004). Asistencia a clase y rendimiento académico en estudiantes de medicina: La experiencia de la Universidad Autónoma de Barcelona. Educación Médica, 7(2), 85-89. Recuperado de http://scielo.isciii. es/pdf/edu/v7n2/original4.pdf

Quispe, J. T. (enero, 2010). Factores que influyen en el rendimiento académico y la deserción de los estudiantes de la Facultad de Ingeniería Económica de la UNA-PUNO, período 2009. Cuadernos de Educación y Desarrollo, 2(11). Recuperado de http://www.eumed. net/rev/ced/11/jtq.htm 